\title{
Editorial
}

\section{Interventional Nephrology: A Big Opportunity for a Big Problem}

\author{
Tushar J. Vachharajani*, ${ }^{*}$

\section{Nephrology Section, W. G. (Bill) Hefner Veterans Affairs Medical Center, Salisbury, NC-28144, USA}

Keywords: Dialysis, hemodialysis, dialysis access, interventional nephrology, vascular access, endovascular procedures, peritoneal dialysis.

A great spiritual teacher from the Indian subcontinent, Siddhartha Gautam "Buddha" said, "There are two mistakes one can make along the road to truth - not starting and not going all the way."

The field of Interventional Nephrology needs to be viewed as a new road towards improving the dialysis access care provided to chronic kidney disease patients across the globe. Dialysis access remains a weak link and a major hurdle both in the planning and maintenance phase of renal replacement therapy. Traditionally the nephrologist has assumed the role of a "thinker" and delegated the procedural aspect of care to surgeons for access creation and radiologists for access maintenance procedures. As a result the dialysis access care is treated like a step-child. The end result of such a fragmented care leads to patient inconvenience and high healthcare cost to the society.

The nephrologist is ideally suited to understand the complexities involved with renal replacement therapy. The advent of Interventional Nephrology in the United States has created a new opportunity for the nephrologists to change their roles from "thinkers" to "doers" and "leaders". The subspecialty is a step towards not only learning the necessary skills to perform dialysis access related procedures but to assume a leadership role in coordinating a team to provide the best possible care. The subspecialty received a further boost with the establishment of the American Society of Diagnostic and Interventional Nephrology (www.ASDIN.org) in 2000.

Over the past decade, the society has succeeded in convincing the medical community about the role of a nephrologist as an interventionalist in learning the skills and performing procedures such as - fistulograms, percutaneous balloon angioplasty, thrombectomies, coil and stent placements, tunneled catheter placements, renal ultrasounds, peritoneal dialysis catheter placements, renal arteriograms and renal biopsies. As a leader the interventional nephrologist can coordinate with the dialysis staff and patients as well as with the

*Address correspondence to this author at the Nephrology Section, W. G. (Bill) Hefner Veterans Affairs Medical Center, Salisbury, NC -28144, USA; Tel: 704638-9000; Fax: 704-638-3855; E-mail: tvachh@gmail.com

$\S$ Guest Editor surgeons and radiologists providing a broad multi-pronged approach to access care. Teamwork divides the task and doubles the success, providing the impetus for ideal dialysis access care.

It is time to expand the benefits reaped by the chronic kidney disease patients in the United States with the growing success of this new subspecialty to the global dialysis community and this special section covers the basics of interventional nephrology.

The scope of basic endovascular procedures performed by nephrologist has been well covered by Niyyar and her colleagues. The nephrologists have been successfully trained and are performing the basic endovascular procedures effectively and safely. The benefit of timely intervention has reduced the number of hospitalizations and the associated morbidity and mortality.

The growth of a peritoneal dialysis program can be directly traced to the level of interest demonstrated by the nephrologist. The multiple techniques of placing peritoneal dialysis catheters are discussed in the next paper by Buffington et al. Finally, Haddad et al. provide an excellent overview of the dreaded complication of central vein stenosis associated with tunneled catheters.

The primary focus of this supplement is to generate interest and awareness of the scope of interventional nephrology. The nephrology community across the globe needs to evaluate the available resources to discuss the role of various team members and strive to improve dialysis access care. The growth and progress of the subspecialty is evident from the increasing number of academic centers in the United States incorporating procedural training as part of the nephrology fellowship [1,2]. The trend is also seen as a recruiting tool to train the nephrology workforce of the future [3].

\section{REFERENCES}

[1] Vachharajani TJ, Moossavi S, Salman L et al. Dialysis vascular access management by interventional nephrology programs at University Medical Centers in the United States. Semin Dial 2011; 24: 564-9.

[2] Vachharajani TJ, Moossavi S, Salman L et al. Successful models of interventional nephrology at academic medical centers. Clin J Am Soc Nephrol 2010; 5: 2130-6.

[3] Parker MG. Nephrology training in the 21st century: toward outcomesbased education. Am J Kidney Dis 2010; 56: 132-42.

(C) Tushar Vachharajani; Licensee Bentham Open.

This is an open access article licensed under the terms of the Creative Commons Attribution Non-Commercial License (http://creativecommons.org/licenses/by$\mathrm{nc} / 3.0 /$ ) which permits unrestricted, non-commercial use, distribution and reproduction in any medium, provided the work is properly cited. 